\title{
Efficacy and safety of tisagenlecleucel in Japanese adult patients with relapsed/refractory diffuse large B-cell lymphoma
}

\author{
Hideki Goto ${ }^{1} \cdot$ Shinichi Makita ${ }^{2} \cdot$ Koji Kato $^{3} \cdot$ Kota Tokushige $^{4} \cdot$ Taizo Fujita $^{4} \cdot$ Koichi Akashi $^{3} \cdot$ Koji Izutsu $^{2}$. \\ Takanori Teshima ${ }^{1}$
}

Received: 3 February 2020 / Accepted: 1 May 2020 / Published online: 24 May 2020

(c) The Author(s) 2020

\begin{abstract}
Background Tisagenlecleucel demonstrated a high rate of durable response in adult patients with relapsed/refractory $(\mathrm{r} / \mathrm{r})$ diffuse large B-cell lymphoma (DLBCL) in the pivotal global phase 2 JULIET study. Here, we report the efficacy and safety of tisagenlecleucel in the Japanese subgroup.

Methods JULIET (NCT02445248) is a single-arm, open-label, multicenter, phase 2 study involving adult patients with $\mathrm{r} / \mathrm{r}$ DLBCL who either relapsed after or were ineligible for autologous stem cell transplant. Primary endpoint was best overall response rate (ORR; complete response $[\mathrm{CR}]+$ partial response $[\mathrm{PR}])$ as judged by an independent review committee.

Results In Japan, of 17 patients enrolled, 9 were infused with tisagenlecleucel and completed $\geq 3$ months of follow-up. Best ORR was $77.8 \%$ (7/9; 95\% confidence interval, 40.0-97.2), with 5 patients (55.6\%) in CR and $2(22.2 \%)$ in PR. Cytokine release syndrome (CRS) occurred in 6 patients (66.7\%), with grade 3 CRS in 2 patients (Penn grading scale). Two patients received tocilizumab. Two deaths (22.2\%) occurred more than 30 days after tisagenlecleucel infusion due to disease progression, neither of which were related to tisagenlecleucel.

Conclusion Tisagenlecleucel showed a high best ORR with a manageable safety profile, thus offering a new treatment option in selected Japanese patients with $\mathrm{r} / \mathrm{r}$ DLBCL.
\end{abstract}

Keywords Tisagenlecleucel $\cdot$ CAR T-cell therapy $\cdot$ Diffuse large B-cell lymphoma $\cdot$ JULIET

\section{Introduction}

Diffuse large B-cell lymphoma (DLBCL) is an aggressive and the most common type of non-Hodgkin lymphoma (NHL) in Japan, with an incidence of 33\% [1]. Survival rates have improved over time with immunochemotherapy combinations containing rituximab. However, nearly $40 \%$ of patients become relapsed/refractory $(\mathrm{r} / \mathrm{r})$ after treatment, are difficult to manage with conventional cytotoxic

Hideki Goto

hidekigt@med.hokudai.ac.jp

1 Department of Hematology, Graduate School of Medicine, Hokkaido University Faculty of Medicine, Sapporo, Japan

2 Department of Hematology, National Cancer Center Hospital, Chuo-ku, Tokyo, Japan

3 Department of Medicine and Biosystemic Science, Kyushu University Graduate School of Medical Sciences, Fukuoka, Japan

4 Novartis Pharma K.K., Toranomon Minato-ku, Tokyo, Japan chemotherapy, and are associated with high treatmentrelated mortality rates [2]. In a retrospective study assessing the outcomes in 636 patients with refractory DLBCL, the overall response rate (ORR) was $26 \%$, with only $7 \%$ of patients achieving a complete response (CR) and $18 \%$ achieving a partial response (PR), with a median overall survival (OS) of 6.3 months [3]. Despite overall improvements in outcomes of DLBCL, $\mathrm{r} / \mathrm{r}$ disease remains a major cause of morbidity and mortality. To improve the outcome of $\mathrm{r} / \mathrm{r}$ DLBCL, several novel therapies are being developed. Among them, anti-CD19 chimeric antigen receptor (CAR) T-cell therapy is considered one of the most promising and effective therapies for $\mathrm{r} / \mathrm{r}$ DLBCL [4].

Tisagenlecleucel is a second-generation anti-CD19 CAR T-cell utilizing 4-1BB as a co-stimulatory domain. It targets CD19+ B cells and has shown efficacy against various subtypes of B-cell lymphomas, including DLBCL, in a singlecenter, phase 2a study [5]. Subsequently, a phase 2, multicenter, global, pivotal trial (JULIET; NCT02445248) was conducted, and tisagenlecleucel demonstrated a high rate 
of durable response in adults with r/r DLBCL [6]. Here, we report a subgroup analysis of the Japanese patients infused with tisagenlecleucel for $\mathrm{r} / \mathrm{r}$ DLBCL in the JULIET trial.

\section{Patients and methods}

\section{Study design and patient population}

The design of the single-arm, open-label, phase 2, multicenter, global trial of tisagenlecleucel (JULIET) has been described previously (Fig. 1) [6]. Eligible patients were 18 years or older with r/r DLBCL who had previously received $\geq 2$ lines of chemotherapy, including rituximab and anthracyclines. The current subgroup analysis included Japanese patients infused with tisagenlecleucel. Patients were excluded if they had previously received CD19-directed therapy or an allogeneic transplant or had primary mediastinal DLBCL or active central nervous system (CNS) involvement by DLBCL. Enrolled patients underwent bridging chemotherapy as needed and a round of lymphodepleting chemotherapy consisting of either fludarabine $25 \mathrm{mg} / \mathrm{m}^{2}$ and cyclophosphamide $250 \mathrm{mg} / \mathrm{m}^{2} /$ day $\times 3$ days or bendamustine $90 \mathrm{mg} / \mathrm{m}^{2} /$ day $\times 2$ days. Autologous leukapheresis products collected and cryopreserved at the clinical sites were shipped to the manufacturing facility (Morris Plains, NJ, USA) for tisagenlecleucel manufacturing, and the concentrated and cryopreserved cells were shipped back to the clinical sites for infusion. Patients received tisagenlecleucel infusion as a single target dose range of $1-5 \times 10^{8} \mathrm{CD} 19$-transduced viable CAR-T cells. The study was sponsored and designed by Novartis Pharmaceuticals Corporation and Novartis Pharma
K.K. and was approved by the institutional review board at each participating institution. All patients provided written informed consent. Data were analyzed and interpreted by the sponsors in collaboration with all authors, and all authors reviewed the manuscript and vouch for the accuracy of the data and analysis.

\section{Endpoints}

The primary endpoint was best ORR $(\mathrm{CR}+\mathrm{PR})$ per independent review committee [6], using the Lugano Classification [7] integrating anatomical imaging and functional imaging with positron emission tomography. Secondary endpoints included the duration of overall response (DOR), progression-free survival (PFS), event-free survival (EFS), OS, safety, and cellular kinetics.

\section{Statistical analysis}

This is a subgroup analysis of all patients in the JULIET trial enrolled from medical institutions situated in Japan. Efficacy and safety analyses included all patients who received tisagenlecleucel infusion, and cellular kinetic analysis included all infused patients who had at least 1 sample providing evaluable cellular kinetic data for tisagenlecleucel. The current subgroup analysis was performed after all Japanese patients infused with tisagenlecleucel had completed at least 3 months of follow-up or were discontinued earlier (data cutoff date: May 21, 2018).

All data analyses were descriptive in nature owing to the small size of the Japanese subgroup. The primary efficacy endpoint of best ORR was reported with the exact binomial

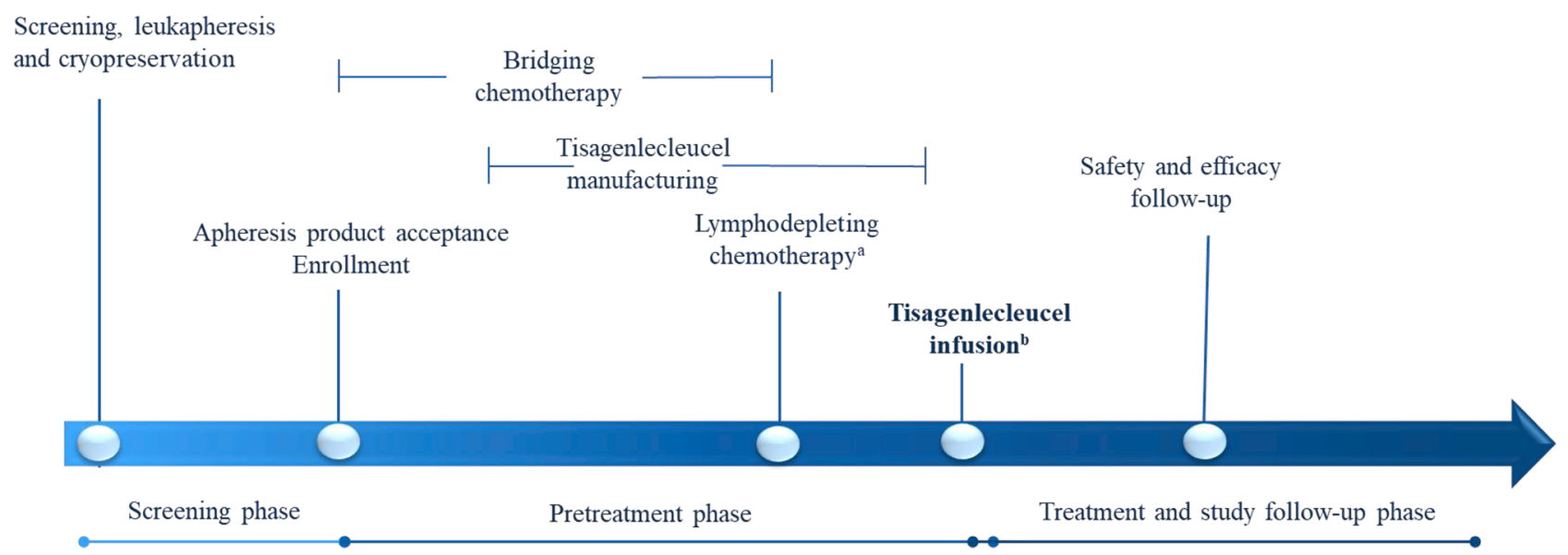

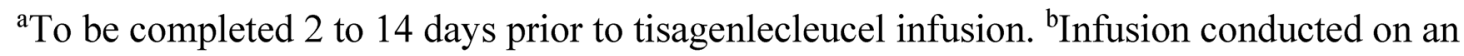
inpatient basis in Japan.

Fig. 1 Study design 
95\% confidence interval (CI). OS was illustrated by best overall response using a swimmer plot, along with relapse/ progression status. For reporting of adverse events (AEs), the Medical Dictionary for Regulatory Activities (MedDRA), version 21.0, and the Common Terminology Criteria for Adverse Events (CTCAE), version 4.03 [8], were used. Specifically, the cytokine release syndrome (CRS) grading was reported according to the Penn grading scale [9]. Individual concentration-time profiles for tisagenlecleucel transgene levels assessed by quantitative polymerase chain reaction (qPCR) in peripheral blood were graphically presented by the month 3 disease response. A complete and detailed description of the overall study design and statistical analysis for the JULIET trial has been published previously [6].

\section{Results}

Seventeen Japanese patients were enrolled, of which 9 were infused with tisagenlecleucel. Eight patients discontinued prior to tisagenlecleucel infusion due to disease progression (physician decision; $n=4$ ), tisagenlecleucel product-related issues $(n=2)$, AEs $(n=1)$, and patient withdrawal $(n=1)$. Study follow-up was still ongoing for 6 patients infused with tisagenlecleucel. Three patients ( 2 deaths, 1 consent withdrawal) discontinued from the study follow-up (Fig. 2).

Of the 9 patients infused with tisagenlecleucel, 7 had relapsed disease and 2 had refractory disease at the start of the trial. The median age of the patients was 61 years, and 4 patients were aged more than 65 years. Four patients had received prior hematopoietic stem cell transplant.
Prior lines of therapy ranged from 2 to 5. The histology review confirmed DLBCL in 7 patients, 1 of whom had transformed mucosa-associated lymphoid tissue lymphoma (Table 1). Bridging chemotherapy was used in six patients, and the treatment regimens included R-EPOCH, C-MOPP, R-ESHAP, R-GDP, and ICE. Overall disease responses prior to tisagenlecleucel infusion assessed by investigator were SDs in three patients, and CR, PR and progressive disease in one patient each. The median dose of tisagenlecleucel infusion was $2.0 \times 10^{8} \mathrm{CAR}$-positive viable $\mathrm{T}$ cells (range, $1.0-4.9 \times 10^{8}$ cells). The median time from enrollment to infusion was 63 days (range, 46-71).

\section{Efficacy}

At data cutoff (May 21, 2018), the best ORR achieved was $77.8 \%$ (95\% CI, 40.0-97.2), with 5 patients (55.6\%) achieving a CR, $2(22.2 \%)$ achieving a PR, and $2(22.2 \%)$ with progressive disease (Table 2). At the month 3 follow-up, 5 patients (55.6\%) were in CR and $1(11.1 \%)$ patient had stable disease. Of the patients evaluated at the 6-month follow-up $(n=4), 2$ patients were in CR. Among the 7 patients with a best response of $\mathrm{CR}$ or $\mathrm{PR}$, the time to response ranged from 28 to 91 days, and the DOR ranged from $1+$ to 523 + days. Among all infused patients, the PFS and EFS ranged from 29 to 550 + days, and the OS ranged from 45 to $710+$ days (Table 2, Fig. 3). The DOR, PFS, EFS, and OS findings need to be interpreted with caution because the data for these endpoints were immature at the time of analysis, with considerably censored observations; therefore, the Kaplan-Meier estimates, such as median time, were not calculated for these endpoints.

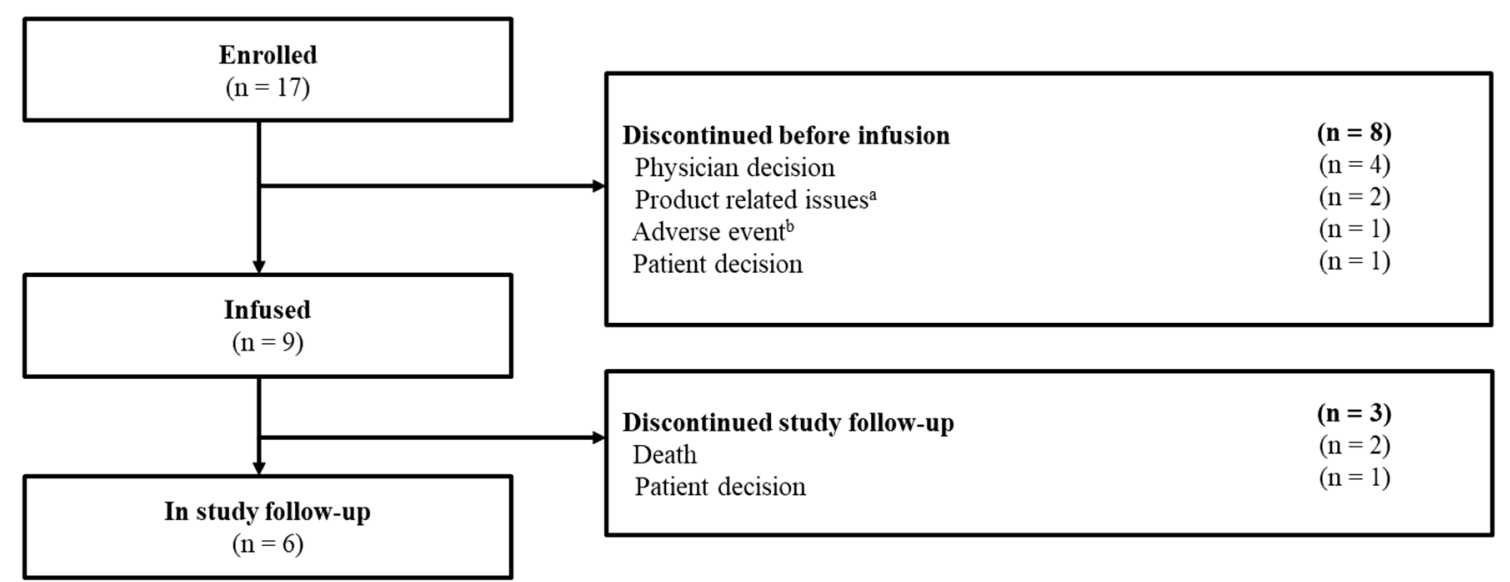

${ }^{a}$ Manufacturing was terminated prior to harvest due to suboptimal cell growth. ${ }^{\text {binfection, }}$ Pneumonitis, Bronchopulmonary aspergillosis, and Sepsis.

Fig. 2 Patient disposition 
Table 1 Patients' demographics (infused patients, $N=9$ )

\begin{tabular}{|c|c|c|c|c|c|c|c|c|c|}
\hline $\begin{array}{l}\text { Baseline character- } \\
\text { istics }\end{array}$ & Patient A & Patient B & Patient C & Patient D & Patient E & Patient $\mathrm{F}$ & Patient G & Patient $\mathrm{H}$ & Patient I \\
\hline Age, years/sex & $45 / \mathrm{F}$ & $65 / \mathrm{M}$ & $73 / \mathrm{F}$ & $41 / \mathrm{M}$ & 69/M & $70 / \mathrm{F}$ & $61 / \mathrm{F}$ & $32 / \mathrm{M}$ & $58 / \mathrm{M}$ \\
\hline Prior HSCT & Yes & No & No & Yes & No & No & Yes & No & Yes \\
\hline Histology review & DLBCL & Other $^{\mathrm{a}}$ & DLBCL & DLBCL & DLBCL & tMALT lymphoma & DLBCL & DLBCL & DLBCL \\
\hline Cell of origin ${ }^{b}$ & $\mathrm{ABC}$ & NA & $\mathrm{ABC}$ & $\mathrm{ABC}$ & GCB & $\mathrm{ABC}$ & GCB & $\mathrm{ABC}$ & $\mathrm{ABC}$ \\
\hline $\begin{array}{l}\text { Previous lines of } \\
\text { therapies }\end{array}$ & 4 & 2 & 3 & 2 & 3 & 5 & 2 & 2 & 3 \\
\hline $\begin{array}{l}\text { Disease status at the } \\
\text { start of the trial }\end{array}$ & Relapsed & Relapsed & Relapsed & Relapsed & Relapsed & Refractory & Relapsed & Relapsed & Refractory \\
\hline Double/triple hits & Negative & NA & Negative & Negative & Negative & Negative & Negative & $\begin{array}{l}M Y C+B C L 6+\text { rear- } \\
\text { rangements }\end{array}$ & NA \\
\hline $\begin{array}{l}\text { Bridging chemo- } \\
\text { therapy prior to } \\
\text { infusion }\end{array}$ & Yes & Yes & No & No & Yes & Yes & Yes & Yes & No \\
\hline $\begin{array}{l}\text { Investigator-assessed } \\
\text { overall response } \\
\text { prior to infusion }\end{array}$ & $\mathrm{CR}$ & SD & $\mathrm{PD}$ & PD & SD & PR & SD & PD & SD \\
\hline $\begin{array}{l}\mathrm{LDH}(\mathrm{U} / \mathrm{L}) \text { prior to } \\
\text { infusion }^{\mathrm{c}}\end{array}$ & 144 & $331 \mathrm{H}$ & 222 & $264 \mathrm{H}$ & $497 \mathrm{H}$ & 202 & $261 \mathrm{H}$ & $510 \mathrm{H}$ & $354 \mathrm{H}$ \\
\hline
\end{tabular}

$A B C$ activated B cell, $D L B C L$ diffuse large B-cell lymphoma, $F$ female, $G C B$ germinal center B cell, $H S C T$ hematopoietic stem cell transplant, $L D H$ lactate dehydrogenase, $M$ male, $N A$ not available, $t M A L T$ transformed mucosa-associated lymphoid tissue

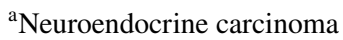

${ }^{\mathrm{b}}$ Choi and modified Choi algorithms and Tally method

${ }^{\mathrm{c}} \mathrm{H}$ denotes a value above the upper limit of normal

Table 2 Overall efficacy and cellular kinetics (infused patients, $N=9$ )

\begin{tabular}{|c|c|c|c|c|c|c|c|c|c|}
\hline Efficacy and cellular kinetic variables & Patient A & Patient B & Patient C & Patient D & Patient E & Patient $\mathrm{F}$ & Patient G & Patient $\mathrm{H}$ & Patient I \\
\hline Best overall response & $\mathrm{CR}$ & PD & $\mathrm{CR}$ & PR & PR & $\mathrm{CR}$ & $\mathrm{CR}$ & $\mathrm{PD}$ & $\mathrm{CR}$ \\
\hline Time to response, days & 28 & - & 28 & 31 & 28 & 30 & 90 & - & 91 \\
\hline Duration of response, days & $523+$ & - & $51+$ & 55 & $52+$ & $148+$ & $1+$ & - & $1+$ \\
\hline Progression-free survival, days & $550+$ & 29 & $78+$ & 85 & $79+$ & $177+$ & $90+$ & 36 & $91+$ \\
\hline Event-free survival, days & $550+$ & 29 & $78+$ & 85 & 126 & 207 & $90+$ & 36 & $91+$ \\
\hline Overall survival, days & $710+$ & 231 & $144+$ & $86+$ & $183+$ & $207+$ & $160+$ & 45 & $91+$ \\
\hline $\mathrm{AUC}_{0-28 \mathrm{~d}}$, copies $/ \mu \mathrm{g} \times$ days & 83,800 & 35,400 & 21,700 & 34,200 & 57,600 & 7290 & 189,000 & $1,740,000$ & 2730 \\
\hline$C_{\max }$, copies $/ \mu \mathrm{g}$ & 9410 & 6120 & 2470 & 4420 & 5840 & 846 & 11,900 & 137,000 & 149 \\
\hline$T_{\max }$, days & 5.82 & 5.85 & 8.78 & 12.9 & 5.73 & 6.96 & 11.0 & 26.7 & 19.8 \\
\hline$T_{1 / 2}$, days & 408 & 7.53 & 26.6 & 1.18 & 32.4 & 4.16 & 77.9 & 2.72 & - \\
\hline
\end{tabular}

$A U C_{0-28 d}$ area under the concentration-time curve from time 0 to day $28, C_{\max }$ maximum concentration, $C R$ complete response, $P D$ progressive disease, $P R$ partial response, $T_{1 / 2}$ half-life, $T_{\max }$ time to maximum expansion, + flags censored observations

\section{Safety}

All 9 patients infused with tisagenlecleucel reported at least 1 AE of any grade severity within 8 weeks of tisagenlecleucel infusion. Grade 3 or grade 4 AEs reported in $\geq 15 \%$ of patients included CRS, anemia, decreased neutrophil count, decreased white blood cell count, decreased lymphocyte count, and decreased platelet count (Table 3). Among the AEs reported between 8 weeks and 1 year of infusion $(n=8)$, the most common ( $\geq 15 \%$ of patients) were nasopharyngitis and decreased white blood cell count, reported in $25 \%$ of patients each. The most common AEs of special interest (AESI) from any cause were cytopenias not resolved by day 28 (77.8\%), CRS (66.7\%), infections (44.4\%), neurological events (11.1\%), and tumor lysis syndrome (11.1\%; Table 4). CRS was reported in 5/7 (71.4\%) patients with disease response and $1 / 2(50.0 \%)$ patients with no response. Grade 2 hypogammaglobulinemia was reported in one patient 
Fig. 3 Overall survival with tisagenlecleucel by best overall response. $C R$ complete response, $D L B C L$ diffuse large B-cell lymphoma, $O S$ overall survival, $P R$ partial response, tMALT transformed mucosaassociated lymphoid tissue lymphoma

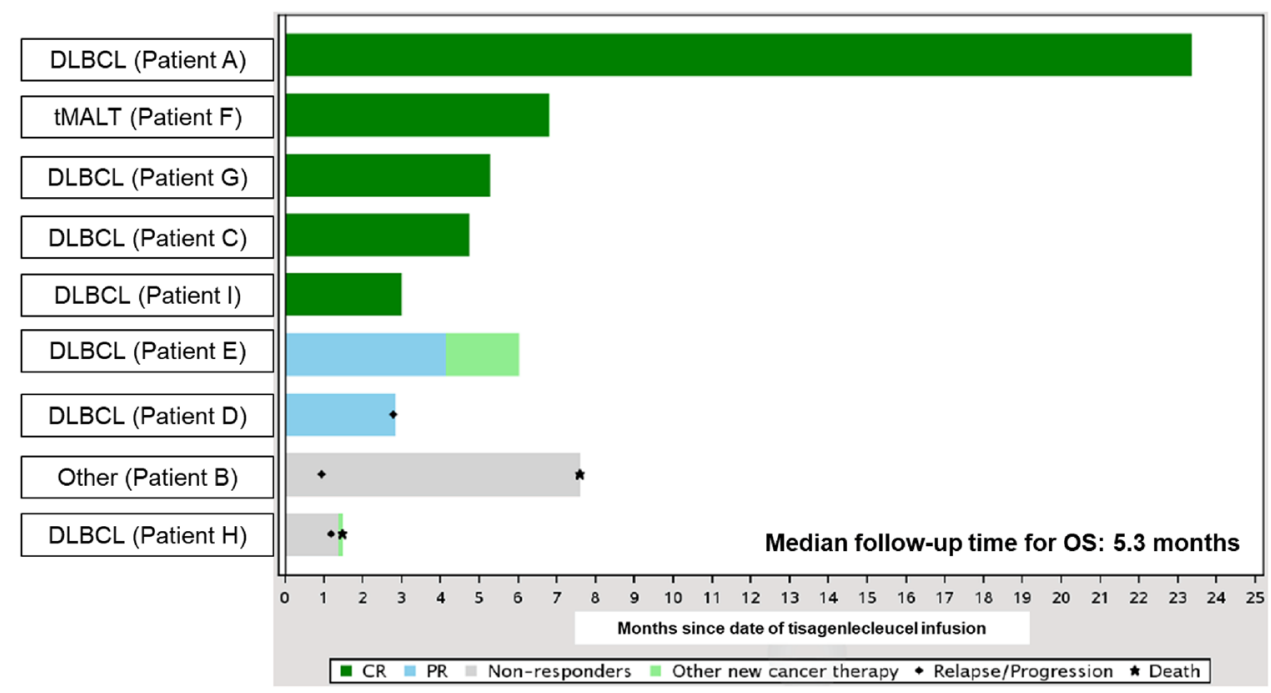

Table 3 Adverse events $\geq 15 \%$, reported within 8 weeks of infusion (infused patients, $N=9)$

Table 4 Adverse events of special interest from any cause ${ }^{\S}$ (infused patients, $N=9$ )

\begin{tabular}{llll}
\hline AEs (regardless of study drug relationship) & \multicolumn{2}{l}{ Patients $(N=9)$} \\
\cline { 2 - 4 } & All grades, $n(\%)$ & Grade 3, $n(\%)$ & Grade 4, $n(\%)$ \\
\hline Cytokine release syndrome & $6(66.7)$ & $2(22.2)$ & 0 \\
Anemia & $4(44.4)$ & $2(22.2)$ & 0 \\
Decreased neutrophil count & $4(44.4)$ & $2(22.2)$ & $2(22.2)$ \\
Decreased white blood cell count & $4(44.4)$ & $2(22.2)$ & $1(11.1)$ \\
Decreased lymphocyte count & $3(33.3)$ & $1(11.1)$ & $2(22.2)$ \\
Decreased platelet count & $3(33.3)$ & $1(11.1)$ & $1(11.1)$ \\
Decreased appetite & $3(33.3)$ & $1(11.1)$ & 0 \\
\hline
\end{tabular}

AEs are based on MedDRA preferred term

$A E$ adverse event, MedDRA Medical Dictionary for Regulatory Activities

\begin{tabular}{llll}
\hline & $\begin{array}{l}\text { Patients }(N=9) \\
\text { All grades, } n(\%)\end{array}$ & Grade 3, $n(\%)$ & Grade 4, $n(\%)$ \\
\hline Cytokine release syndrome $^{\mathrm{a}}$ & $6(66.7)$ & $1(11.1)$ & $1(11.1)$ \\
Neurological events $^{\mathrm{b}}$ & $1(11.1)$ & $1(11.1)$ & 0 \\
Cytopenias not resolved by day 28 & $7(77.8)$ & $6(66.7)$ & $1(11.1)$ \\
Infections & $4(44.4)$ & 0 & 0 \\
Tumor lysis syndrome & $1(11.1)$ & 0 & $1(11.1)$ \\
\hline
\end{tabular}

AESI adverse event of special interest, MedDRA Medical Dictionary for Regulatory Activities

${ }^{\S}$ Occurring within 8 weeks of tisagenlecleucel infusion

${ }^{\mathrm{a}}$ Cytokine release syndrome was graded using the Penn grading scale

${ }^{\mathrm{b}}$ The neurological event reported in 1 patient was delirium. AESIs are based on grouped term consisting of MedDRA preferred term(s) only, who received intravenous immunoglobulin (IVIG) four times from day 30 to day 149 (follow-up is ongoing). In total, four patients received IVIG after tisagenlecleucel infusion, which include three patients receiving IVIG since before tisagenlecleucel infusion. None of the four patients developed severe infection. Grade 3 and grade 4 cytopenias were reported in 6 patients (66.7\%) and 1 patient (11.1\%), respectively. In the patient who experienced grade 4 cytopenias, thrombocytopenia continued since before tisagenlecleucel infusion, worsened to grade 4 on day 19 , and recovered to grade 3 on day 279 (after the data cutoff date). The patient received multiple platelet transfusions before 
and after tisagenlecleucel infusion. Grade 3 and grade 4 CRS were reported in 1 patient each (11.1\%). Among the 6 patients who reported CRS, 3 patients required oxygen supplementation and 2 patients required anti-cytokine therapy with tocilizumab. The median time to onset of CRS was 4 days (range, 1-8; Table 5). Neurological event occurred in one patient, who developed grade 1 delirium on day 1 , worsened to grade 3 on day 7 and resolved on the same day. Two deaths were reported due to disease progression more

Table 5 Cytokine release syndrome

\begin{tabular}{ll}
\hline & $\begin{array}{l}\text { Patients with } \\
\text { CRS }(N=6)\end{array}$ \\
\hline Time to onset, median (range), days & $4.0(1-8)$ \\
Duration, median (range), days & $7.5(4-11)$ \\
Oxygen supplementation, $n(\%)$ & $3(50.0)$ \\
Anti-cytokine therapy, $n(\%)$ & $2(33.3)$ \\
Tocilizumab & $2(33.3)$ \\
Corticosteroids & 0 \\
ICU admission, $n(\%)$ & $0^{\mathrm{a}}$ \\
Hypotension that required intervention, $n(\%)$ & 0 \\
High-dose vasopressors & 0 \\
Intubated, $n(\%)$ & 0 \\
\hline
\end{tabular}

$C R S$ cytokine release syndrome, $H L H$ hemophagocytic lymphohistiocytosis, ICU intensive care unit, TLS tumor lysis syndrome

${ }^{a}$ One patient, who developed CRS (day 8-11), HLH (day 10-42), and TLS (day 11-32), and admitted to the ICU (day 12-18), is not included than 30 days after infusion: 1 patient died due to DLBCL and the other patient died due to his primary disease of neuroendocrine carcinoma.

\section{Cellular kinetics}

Concentration-time profiles of tisagenlecleucel were generally similar between patients who had a month 3 response and those who did not (Fig. 4). Similar expansion, measured as maximum concentration $\left(C_{\max }\right)$ and area under the concentration-time curve from time 0 to day 28 $\left(\mathrm{AUC}_{0-28 \mathrm{~d}}\right.$ ), was also observed in patients regardless of the response, with high variability in the transgene levels (Table 2). Thus, no apparent effect of exposure on clinical outcome was observed.

\section{Discussion}

The JULIET trial is a phase 2, multicenter, global, pivotal trial of tisagenlecleucel, a second-generation antiCD19 CAR T-cell therapy [6]. This subgroup analysis of JULIET assessed the efficacy, safety, and cellular kinetic profile of tisagenlecleucel in Japanese adult patients with $\mathrm{r} / \mathrm{r}$ DLBCL. To our knowledge, this is the first report of an anti-CD19 CAR T-cell therapy in a Japanese subgroup to address the poor prognosis in this patient population. Tisagenlecleucel demonstrated substantial efficacy

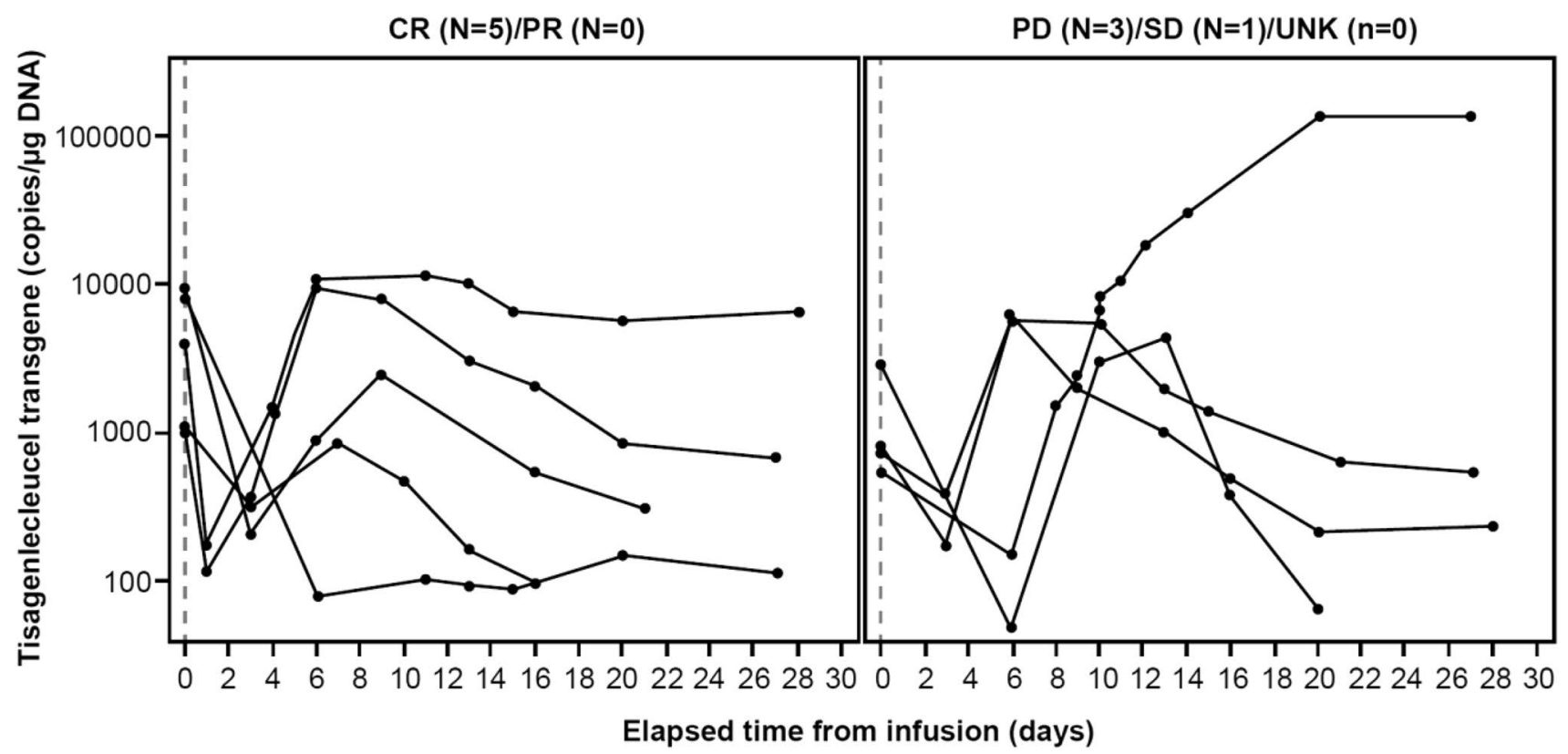

Fig. 4 Tisagenlecleucel transgene levels assessed by qPCR up to month 1 in peripheral blood, by month 3 response as judged by the independent review committee. $C R$ complete response, DNA deoxy- ribonucleic acid, $P D$ progressive disease, $P R$ partial response, $q P C R$ quantitative polymerase chain reaction, $S D$ stable disease, $U N K$ unknown 
in selected Japanese patients with $\mathrm{r} / \mathrm{r}$ DLBCL. The best ORR achieved was $77.8 \%$ (7/9; 95\% CI, 40.0-97.2) with 5 CRs. Four out of 7 responders had an ongoing response at the time of data cutoff (median follow-up time for DOR and OS, 1.7 and 5.3 months, respectively), including 1 patient with a CR lasting for more than 17 months. One patient who had transformed mucosa-associated lymphoid tissue lymphoma achieved a CR with tisagenlecleucel. In the JULIET study $(n=93)$, tisagenlecleucel demonstrated durable responses, with a best ORR of 52\%. The response rates at month 3 and month 6 were $38 \%$ and 33\%, respectively [6]. Overall, efficacy was consistent between the overall and Japanese population. However, results from the Japanese subgroup should be interpreted with caution owing to the small number of patients.

Similar to tisagenlecleucel, high response rates with durable responses have also been observed with other antiCD19 CAR T-cell therapies in the ZUMA-1 and TRANSCEND-NHL-001 trials $[10,11]$. This confirms the role of anti-CD19 CAR T-cells for the treatment of $\mathrm{r} / \mathrm{r}$ aggressive B-cell NHL. The overall JULIET study confirmed the durable ongoing responses in patients with $\mathrm{r} / \mathrm{r}$ DLBCL who achieved a response (CR or PR) [6], suggesting possible sustained efficacy of tisagenlecleucel in the Japanese patients who achieved a response.

The relationships between several biomarkers and efficacy of tisagenlecleucel were assessed in the overall JULIET study, and multivariate analyses identified that the high levels of LDH, a known marker of tumor burden and disease aggressiveness, at pre-infusion were associated with poor efficacy outcomes [12]. In our subgroup analysis, two non-responders and four responders also had high LDH levels at pre-infusion (Table 1), showing that high levels of LDH at pre-infusion might not necessarily cause insufficient response in Japanese population.

In Japan, hospitalization for safety monitoring is required from initiation of lymphodepleting chemotherapy until 3 weeks after infusion, while patients could receive infusions in either inpatient or outpatient settings outside Japan. The safety profile of tisagenlecleucel in the Japanese patients was consistent with that in the overall population (CRS, 58\%) [6]. CRS was reported in $67 \%$ of the Japanese patients. All CRS was resolved within two weeks and was manageable with appropriately trained study-site personnel. No deaths were attributed to tisagenlecleucel, CRS, or cerebral edema. The 2 deaths reported in the Japanese subgroup occurred due to disease progression more than 30 days after tisagenlecleucel infusion. In the JULIET trial, CRS was graded using the Penn grading scale and was managed with tocilizumab as per protocolspecific algorithm [6], which included administration of anti-cytokine therapy for patients who did not respond to supportive care. The cellular kinetics of tisagenlecleucel observed in the Japanese subgroup were consistent with that observed in the overall population despite high variability [6]. Eight patients did not receive infusion mainly due to physician decision of disease progression in 4 patients after leukapheresis, suggesting the importance of disease control during the manufacturing process and patient selection. The dropout rate in Japanese patients is similar to that in the overall JULIET study, and the high dropout rate may be associated with a long turn-around time. It might be an important challenge in the future to shorten the turn-around time of CAR T-cell therapy to improve the accessibility of CAR T-cell therapy in Japan. Bridging chemotherapy was used to maintain optimal health during the manufacturing process as needed. Before tisagenlecleucel infusion, overall disease response was assessed by investigator, and CR was achieved in one patient. After tisagenlecleucel infusion, the response continued more than 523 days, which highlights the importance of further investigation on optimal bridging chemotherapy for achieving durable response.

There are two other Japanese patients to be highlighted. Manufactured product for one patient did not meet a release criterion of cell viability, which is out of specification (OOS). The OOS product was provided to the patient through an exceptional release procedure. The procedure includes assessment of a product quality, efficacy and safety risk performed by the study sponsor, followed by assessment of risk/benefit by the investigator, confirming that the use of OOS product provides a potential patient benefit that outweighs the disease to be treated with the OOS product. Informed consent for the release of OOS product was obtained from the patient. No severe AE was reported after tisagenlecleucel infusion, and CR was achieved. However, the appropriateness of providing OOS product should be evaluated on a case-by-case basis in the future studies. One patient with DLBCL fulfilling the enrollment criteria was enrolled into this study but had a relapse in lung several months prior to the study entry. This relapse was retrospectively diagnosed as neuroendocrine carcinoma a few months after tisagenlecleucel infusion. It was not clinically feasible to perform biopsy on this patient prior to enrollment and this deviation may represent a challenge in selecting patients for tisagenlecleucel treatment in clinical practice. The present subgroup analysis has several limitations, including the limited number of patients; a high rate of dropouts after enrollment, which may be associated with a long turn-around time; and a relatively short follow-up duration. To confirm the actual role of tisagenlecleucel in Japan, further evaluation in a larger number of Japanese patients with a sufficient follow-up duration is required.

Despite several limitations, the results of this subgroup analysis support the use of tisagenlecleucel in Japanese patients with $\mathrm{r} / \mathrm{r}$ DLBCL who have no effective treatment 
options. The high response rate and manageable safety profile observed with tisagenlecleucel are promising and offer a potential new treatment option for Japanese adult patients with r/r CD19-positive DLBCL.

Acknowledgements We thank the patients and their families, the study investigators, and the study-site personnel for their participation and contribution to this study. We thank Kazuto Natsume for analyzing and interpreting the cellular kinetics data. We thank Rama Mylapuram (Novartis Healthcare Pvt. Ltd.) for medical editorial assistance with this manuscript.

Author contributions $\mathrm{HG}, \mathrm{SM}, \mathrm{KK}, \mathrm{KA}, \mathrm{KI}$, and TT enrolled and treated patients, and gathered data. KT analyzed data. All authors interpreted the data, and participated in writing the article, provided feedback throughout the development process, and approved the final submitted version.

Funding This study was sponsored by Novartis Pharmaceuticals Corporation and Novartis Pharma K.K.

\section{Compliance with ethical standards}

Conflict of interest S.M. received personal fees from Novartis, Takeda, Eisai, Daiichi Sankyo, and Celgene. K.A. reports research grants from Novartis, MSD, Asahi Kasei Pharma, Astellas, AbbVie, Alexion, Chemo-Sero-Therapeutic Research Institute, Japan Blood Products Organization, Eisai, Otsuka, Ono, Yakult, Shin Nippon Biomedical Laboratories, Kyowa Hakko Kirin, Sanofi, Shionogi, Daiichi Sankyo, Taisho, Dainippon Sumitomo, Taiho, Takeda, Mitsubishi Tanabe, Chugai, Teijin, FUJIFILM Toyoma Chemical, Eli Lilly, Nippon Kayaku, Bayer, Bristol-Myers Squibb, Mundipharma, Merck, Mochida, and Nihon Pharmaceutical, and personal fees from Novartis, CSL Behring, MSD, Asahi Kasei Pharma, Astellas Amgen, Astellas, AbbVie, Alexion, Eisai, Otsuka, Ono, Medical Review, Kyowa Hakko Kirin, Sanofi, Shionogi, Shire Japan, SymBio, Celgene, Daiichi Sankyo, Taisho, Dainippon Sumitomo, Takeda, Mitsubishi Tanabe, Chugai, Teijin, Nippon Shinyaku, Eli Lilly, Bayer, Pfizer, Bristol-Myers Squibb, Mundi Pharma, Mochida, and Janssen. K.I. reports research grants from Novartis, Eisai, Kyowa Hakko Kirin, MSD, Takeda, Janssen, Mundipharma, Chugai, AbbVie, Bayer, Ono, Gilead, Zenyaku, Celgene, Solasia, Symbio, Astellas, Astellas Amgen, and Daiichi Sankyo, and personal fees from Kyowa Hakko Kirin, MSD, Takeda, Janssen, Bristol-Myers Squibb, Dainippon Sumitomo, Mundipharma, Nihon Mediphysics, Chugai, Astra Zeneca, AbbVie, Bayer, Ono, and Celgene. T.T. reports research grants and personal fees from Novartis. K.T. and T.F. are employees of Novartis. All other authors declare no competing interests.

Open Access This article is licensed under a Creative Commons Attribution 4.0 International License, which permits use, sharing, adaptation, distribution and reproduction in any medium or format, as long as you give appropriate credit to the original author(s) and the source, provide a link to the Creative Commons licence, and indicate if changes were made. The images or other third party material in this article are included in the article's Creative Commons licence, unless indicated otherwise in a credit line to the material. If material is not included in the article's Creative Commons licence and your intended use is not permitted by statutory regulation or exceeds the permitted use, you will need to obtain permission directly from the copyright holder. To view a copy of this licence, visit http://creativecommons.org/licenses/by/4.0/.

\section{References}

1. Aoki R, Karube K, Sugita Y et al (2008) Distribution of malignant lymphoma in Japan: analysis of 2260 cases, 2001-2006. Pathol Int 58:174-182

2. Sehn LH, Gascoyne RD (2015) Diffuse large B-cell lymphoma: optimizing outcome in the context of clinical and biologic heterogeneity. Blood 125:22-32

3. Crump M, Neelapu SS, Farooq U et al (2017) Outcomes in refractory diffuse large B-cell lymphoma: results from the international SCHOLAR-1 study. Blood 130:1800-1808

4. Makita S, Yoshimura K, Tobinai K (2017) Clinical development of anti-CD19 chimeric antigen receptor T-cell therapy for B-cell non-Hodgkin lymphoma. Cancer Sci 108:1109-1118

5. Schuster SJ, Svoboda J, Chong EA et al (2017) Chimeric antigen receptor T cells in refractory B-cell lymphomas. N Engl J Med 377:2545-2554

6. Schuster SJ, Bishop MR, Tam CS et al (2019) Tisagenlecleucel in adult relapsed or refractory diffuse large B-cell lymphoma. $\mathrm{N}$ Engl J Med 380:45-56

7. Cheson BD, Pfistner B, Juweid ME et al (2007) Revised response criteria for malignant lymphoma. J Clin Oncol 25:579-586

8. U.S. Department for Health and Human Services (2010) Common Terminology Criteria for Adverse Events v4.03. https://evs. nci.nih.gov/ftp1/CTCAE/CTCAE_4.03/CTCAE_4.03_2010-0614_QuickReference_5x7.pdf. Accessed Jan 2019

9. Porter DL, Hwang WT, Frey NV et al (2015) Chimeric antigen receptor $\mathrm{T}$ cells persist and induce sustained remissions in relapsed refractory chronic lymphocytic leukemia. Sci Transl Med 7:303ra139

10. Abramson JS, Gordon LI, Palomba ML et al (2018) Updated safety and long term clinical outcomes in TRANSCEND NHL 001, pivotal trial of lisocabtagene maraleucel (JCAR017) in R/R aggressive NHL. J Clin Oncol 36:7505

11. Neelapu SS, Locke FL, Bartlett NL et al (2017) Axicabtagene ciloleucel CAR T-cell therapy in refractory large B-cell lymphoma. N Engl J Med 377:2531-2544

12. Westin JR, Tam CS, Borchmann P et al (2019) Correlative analyses of patient and clinical characteristics associated with efficacy in tisagenlecleucel-treated relapsed/refractory diffuse large b-cell lymphoma patients in the Juliet trial. Blood 134(supplement_1):4103

Publisher's Note Springer Nature remains neutral with regard to jurisdictional claims in published maps and institutional affiliations. 